\title{
SFA vs. DEA for measuring healthcare efficiency: A systematic review
}

\author{
George Katharakis ${ }^{a\left({ }^{(}\right)}$, Theofanis Katostaras ${ }^{b}$ \\ ${ }^{\text {a }}$ Ph.D. Candidate National and Kapodistrian University, Athens, Greece \\ ${ }^{\mathrm{b}}$ Associate Professor, Laboratory of Clinical Epidemiology, Faculty of Nursing, University of Athens, Greece
}

(*) Corresponding author:

George Katharakis, Ph.D Candidate,

Faculty of Nursing,

National and Kapodistrian University of Athens, Greece,

Address: 123 Papadiamantopoulou, Athens

Tel/Fax: +302107461598

Email: gkathar@nurs.uoa.gr

\begin{abstract}
Frontier techniques have been used to measure healthcare provider efficiency in hundreds of published studies. Although these methods have the potential to be useful to decision makers, their utility is limited by both methodological questions concerning their application. The aim of this paper is to search articles applying combined data envelopment analysis (DEA) and stochastic frontier analysis (SFA) in order to facilitate a common understanding about the adequacy of these methods, defining any differences in healthcare efficiency estimation and the reasons that are behind this. A systematic review of 21 such studies published the last decade was conducted. Only studies written in English were considered. Results are summarized in a form of meta-analysis in order to synthesize results and draw out further implications. Overall, DEA and SFA were found to yield divergent efficiency estimates due to many factors such as statistical noise, how inputs and outputs were defined, as well as data availability. Researchers, besides the combination of models to measure efficiency, lately have introduced environmental variables in their analyses, aiming at better understanding the relationship of these factors to efficiency and thus achieving a better decision making process. In any case the analysis concludes that there is a need for careful attention by stakeholders since the nature of the data and its availability influence the measurement of efficiency and thus it is necessary to model the behavior which generates the data by choosing the appropriate mathematical form.
\end{abstract}

Keywords: Efficiency, Data Envelopment Analysis (DEA), Stochastic Frontier Analysis (SFA), healthcare, systematic review.

\section{Introduction}

Healthcare costs in most developed economies have grown dramatically over the last few decades and it is widely believed that the inefficiency of healthcare institutions, at least in part, has contributed. In response to this belief, an extensive body of literature has addressed the empirical measurement of efficiency in healthcare 
institutions around the world. Hospitals, nursing homes, health maintenance organizations and district health authorities have been the subject of most of these efficiency studies to date. These studies share a common focus; namely, the growing volume of healthcare costs, the effect of these costs on public expenditure and private industry, and the impact of increased competition in the healthcare market. In recent decades healthcare expenditures have grown very significantly in most developed countries, from 6\% of GDP in 1970 to around 11\% of GDP in 2012. Many factors are behind this trend, including a demand for healthcare services that increases with income, and supply factors related with the impact of technological change [1]. This trend might hold in the future or even become more pronounced, due to, among other reasons, the phenomenon of population ageing [2]. The control of healthcare expenditure or its financing are therefore priority aspects when public policies are designed. This priority has increased in recent years given the need to ensure the success of the fiscal consolidation processes in which most economies are immersed, following the surge in budget deficits and public debt during the economic crisis. Among the various economic policy options, those geared to attaining higher levels of efficiency in the provision of health services might be particularly appropriate. This is because, by definition, they would contribute to containing public spending (using fewer resources) while maintaining the same output and quality of the services.

These concerns have created tremendous pressure to measure the efficiency of healthcare providers and systems so that it can be evaluated and improved [3]. Despite widespread interest in evaluating efficiency, considerable uncertainty exists about whether the methods are sufficiently well developed to be used outside the research laboratory [4]. First, the term efficiency is used by different stakeholders to connote various constructs. Second, little is known about how well available efficiency metrics capture the constructs of interest [5]. Payers and purchasers have begun to use efficiency measures despite these uncertainties. Proponents of efficiency measurement seek to "learn on the job" and improve measurements through use. Those who are being evaluated on these metrics worry that the lack of conceptual clarity and the limited methodological assessments increase the likelihood that results from the metrics will create distortions in patterns of care seeking and service delivery, adding to distortions related to current payment systems [6].

The more commonly used techniques are data envelopment analysis (DEA) and stochastic frontier analysis (SFA) which employ quite distinct methodologies for frontier estimation and efficiency measurement, each with associated strengths and weaknesses. Therefore, "...non-statistical approaches such as DEA have the disadvantage of assuming no statistical noise, but have the advantage of being nonparametric and requiring few assumptions about the underlying technology. SFA models on the other hand have the attraction of allowing for statistical noise, but have the disadvantage of requiring strong assumptions as to the form of the frontier" [7]. DEA is favored where measurement error is unlikely to pose much of a threat and where the assumptions of neoclassical production theory are in question. Conversely, SFA should have the advantage in coping with severe measurement error and where simple functional forms provide a close match to the properties of the underlying production technology. Gong and Sickles [8] report findings along similar lines so that "...as misspecification of functional form becomes more serious, DEA's appeal (vis-à-vis SFA) becomes more compelling" [8].

The aim of this systematic review was to identify and critically review the differences of existing applications of frontier techniques that have been used to measure healthcare efficiency. The review is restricted to comparisons between data 
envelopment analysis (DEA) and the most commonly employed parametric alternative, stochastic frontier analysis (SFA). The limitations of the empirical estimation of the efficiency measurement from the application of DEA and SFA are examined. Moreover, this paper is intended to create a common understanding among healthcare stakeholders about the adequacy of these tools to measure healthcare efficiency.

The rest of the paper is structured as follows: Section 2 provides an overview of DEA and SFA techniques for efficiency measurement. Section 3 presents the search procedure along with the typology introduced to classify the articles included in the review. Continuously, Section 3 presents the core results and outlines the findings of the current systematic review. Section 4 and 5 provide a discussion, draw some conclusions and provide key limitations of the current study, with the final section concluding the paper.

\section{Background}

In many of the efficiency analysis studies, researchers have been interested in explaining differences in estimated efficiencies across firms or decision making units (DMU). For the efficiency analysis of DMUs, researchers have applied frontier methods such as DEA or SFA. Both methods measure inefficiency of a DMU as the distance between a best practice (or efficient) frontier and actual performance of the DMU. However, the two methods differ in some key theoretical aspects. DEA measures efficiency relative to a nonparametric estimate of an unobserved true frontier, conditional on observed data [9]. As a nonparametric method, DEA requires no assumptions about the specific form of the frontier or the probability density of inputs and outputs used in the production process. However, DEA assumes no errors and deviations from the efficient frontier rather they are entirely assumed to be due to inefficiency. Stochastic frontier models avoid some of the limitations of the DEA. Specifically, the stochastic methods allow the decomposition of deviations from the efficient frontier into a random error term that embodies statistical noise and a onesided error term representing inefficiency. However, SFA requires the specification of a functional form for the frontier and assumptions about the distributions of the random error and inefficiency error terms which might be very restrictive [10].

DEA measures cost efficiency in two steps. First, given input prices and output levels, the cost-minimizing input vector for each hospital is calculated using linear programming. Next, cost efficiency is measured as the ratio of minimum cost to observed cost and takes a value between 0 and 1, where the value of 1 indicates a cost efficient hospital (for technical details of cost efficiency estimation [11]. The cost efficiency measures the factor by which the observed cost can be reduced if the hospital selects the optimal input bundle (which minimizes the cost of producing a given level of output given input prices) and operates at a technically efficient point (where output is produced using minimum quantities of inputs). Alternatively, cost efficiency can be estimated using SFA which, in a general form, specifies total cost as a function of outputs and input prices plus a composite error term [11]:

Equation 1. Shows here

where $T C_{i}$ represents the total cost of the $i$-th hospital, $Y_{i}$ is a vector of outputs, $W_{i}$ is a vector of input prices, and $\varepsilon_{i}$ is a composite error term which can be calculated with the formula:

Equation 2. Shows here 
where $v_{i}$ captures random statistical noise, assuming that it is normally distributed, and $u_{i}$ represents cost inefficiency for which a distribution (distributions assumed for the one-sided error term: half-normal, truncated-normal, exponential and gamma) must also be assumed. Given the distributional assumptions for the two error terms, the model is estimated by maximum likelihood [11]. In a cross sectional stochastic frontier model, the cost inefficiency is observed indirectly from the estimates of the composite error and is calculated as the expected value of inefficiency, conditional upon the composite residual. In the estimation of a stochastic frontier cost model, one must also specify a functional form for the cost equation. The most popular functional forms used in empirical research have been the translog and CobbDouglas cost functions. The translog function has been shown to be more flexible in the sense that it can provide a second-order differential approximation to any arbitrary function at a single point, making it the preferred functional form in empirical research. However, increased flexibility of the translog function comes at the cost of an increased number of parameters to estimate, and this may give rise to multicolinearity problems [11].

Figure 1. Shows here

From a policy perspective, hospital managers and policymakers can become more effective decision makers by understanding the relationships between efficiency and these two methods. The difference between DEA and SFA is described in Figure 1. Due to measurement error and other random factors affecting output, the stochastic frontier may differ from the best practice DEA frontier. For example, if the error is negative, the stochastic frontier will lie below the deterministic frontier. Using DEA we measure inefficiency as the distance from the estimated production function $f(x)$ and the $x$ produced by $x_{i}$ inputs, (measured by the angled line). Using SFA, the estimated frontier lies below and the distance from it to $x$ is shorter (measured by the curved line). In this case, DEA will result in a higher estimate of inefficiency. Deviations from the production frontier are due to inefficiency. With SFA however, deviations may also arise from a stochastic error. The distance between the DEA frontier and the SFA frontier represents this stochastic error. If the error is positive, the stochastic frontier will lie above the DEA best practice frontier, and DEA will result in lower estimates of inefficiency (higher efficiency).

\section{Materials and Methods}

\subsection{Data Collection Process}

During the last twenty years, non-parametric and parametric methods have been increasingly employed to measure and analyze the productive performance of healthcare services. The healthcare sector is a unique area of application, and one in which the measurement of efficiency has burgeoned over the past few years. Mortimer [7] highlighted the need for parallel application of competing methods for frontier estimation and efficiency measurement. Thus, the set of pair-wise comparisons is steadily growing as new methods for frontier estimation and efficiency measurement arise to address the shortcomings of more traditional methods. In recognition of this fact, the systematic review is restricted to comparisons between data envelopment analysis (DEA) and the most commonly employed parametric alternative stochastic frontier analysis (SFA).

The review is based on literature identified from an initial search of citation databases, review article bibliographies, and web-based resources. Following 
Worthington [12] and Hollingsworth [13] previous works and by adopting the steps and criteria that are summarized in figure 2, only 21 papers, both published and working papers, were included in the review. This sample of papers, all conclude in reporting at least one pair-wise empirical comparison between DEA and SFA during the last decade 2001-2012. It should be noted that the initial search was for the period 1990-2012, but the 21 articles that were kept all refer to the last decade since the key criteria of the search was the comparison approach of DEA and SFA on efficiency measurement. The purpose is to emphasize the fact that either there is progress in the section of controlling these techniques to measure health efficiency or these studies share the same common focus from previously analyzed.

Figure 2. Shows here

The review that was initially started found studies for the period 1990-2012 and exclusively referred to healthcare and hospital efficiency. This paper encompasses a systematic search of all available databases, using Science Direct and Medline. Searches were conducted using the following search terms: 'stochastic frontier', 'frontier estimation', 'frontier techniques'. The above search terms were combined with the term 'data envelopment analysis' and 'hospital efficiency' to further focus the search, yielding 230 articles after the removal of duplicates. References were also used from these studies to identify other relevant articles.

Papers were included if they:

- described applications of both DEA and SFA for measurement hospital efficiency.

- were original or working papers.

- reported data about the reliability and/or validity of the used frontier methods.

Likewise, papers were excluded from the review:

- if they were published in a language other than English.

- if they were abstracts and not full papers.

- if they were technical reports or reviews.

By adopting these criteria and considering that the majority of the published papers that combined both DEA and SFA methods were published in the period of 20002012, 21 articles were identified reporting at least one pair-wise empirical comparison between both DEA and SFA. Thus, the review was focused on articles of this period by aiming to locate results of combinations of frontier techniques to evaluate healthcare efficiency.

\subsection{Classification of relevant studies}

Following the three steps of Worthington [12] and the criteria of Hussey et al. [14] and Mortimer [7] in their reviews, a typology to characterize the papers abstracted in the systematic review was created. This common process, as depicted in Figure 3, forms a convenient framework for the following review.

Figure 3. Shows here 
The introduced typology has four (4) levels:

- Perspective: what is the objective of the evaluation?

- Efficiency measurement approach: what mathematical approach is used?

- Inputs and Outputs: what inputs are used to produce the output included in the measurement?

- Differences in efficiency results: which is the added value of a combination of both DEA and SFA?

The first tier in the typology, perspective, requires an explicit identification of the objective or rationale for the assessment. According to Hussey et al. [14], this element in line with the entity that is evaluating efficiency and the entity that is being evaluated, is important because different entities have different objectives for considering efficiency, have control over a particular set of resources or inputs, and may seek to deliver or purchase a different set of services.

The second tier in the typology, efficient measurement approach, involves describing the mathematical approach used. With regard to the DEA model an identification of the orientation - input or output - and Return to Scale (RTS) is required, while regarding the SFA model the mathematical form and frontier is identified. With regard to the frontier techniques, measures according to several aspects of the methodology used were characterized. For example, the type of data source, the explanatory variables included and the "scientific soundness" (reliability and/or validity) of each measure, was searched.

The third tier in the typology consists of the identification of inputs and outputs used. Outputs identify the outputs of interest and how they are measured. Two types of outputs exist: health services (e.g., visits, admissions and laboratory tests) and health outcomes (e.g., preventable deaths and clinical outcomes such as blood pressure control). On the other hand, inputs are used to produce the outputs of interest that are measured. Inputs can be measured as counts by type (e.g., number of medical staff and number of beds) or they can be monetized. The way in which inputs are measured (physical or financial) influence the way the results are interpreted. Efficiency measures that count physical inputs help to answer questions about whether the output could be produced with fewer people or fewer supplies. Efficiency measures that use financial inputs help to answer questions about whether the output could be produced less expensively, whether the total cost of labor, supplies, and other capital could be reduced through more efficient use or substitution of less costly inputs.

Finally, the fourth tier of the typology identifies the differences of efficiency results derived from DEA and SFA analysis and the factors thought to be associated with these differences.

Thus, the papers were classified by perspective, inputs and outputs, frontier methods used, and scientific soundness.

\section{Results}

\subsection{Overview of studies identified}


The total number of studies identified up to and including 2012 is over 230 . There is a rapid increase in studies over recent years, with almost $80 \%$ of studies having been reported in the last 10 years (Figure 4). This could be explained by the fact that the economic and financial crisis has revealed the need for efficiency measurement both in the public and private sectors.

Figure 4. Shows here

The 21 articles included in the review are presented in Table 1 in the Appendix. $42.86 \%$ of these studies are from United States followed by UK at $14.29 \%$, while the rest of the papers are from Europe, Australia and Canada (Figure 5); the majority of the articles are simulation studies that use quite large samples of healthcare units and the range of their data cover a period of 1,2 or 3 years with the exception of some that use 9 or 11 years of data of inputs and outputs.

Figure 5. Shows here

Figure 6 shows the distribution of the reviewed articles towards the tier of perspective, so that over $55 \%$ of the studies are in the area of decision making and the rest are in the area of research. A significant number of studies (around 10\%) investigate the extension of the used models in measuring the efficiency of healthcare units. The results reflect the intention of using such methods to assess the effectiveness of health units and health services in order to enhance the decision making process.

Figure 6. Shows here

Figure 7 and 8 show that $47.62 \%$ of the studies measured health services as output variables, such as in-patient days or discharges, while inputs were measured in monetary units, indicating the objective of the researcher to answer questions regarding the less expensive production of the outputs. Continuously, $4.76 \%$ of the studies use outcome measures examining changes in health status of individuals treated, while the rest of the studies used both health services and outcome measures. Input variables are mainly measures of staff and capital employed, as examined in about $67 \%$ of the studies. Some of the studies use environmental variables for better results in the measurement of the efficiency scores. Numerous classes of these factors may influence measured levels of organizational attainment. These include: differences in the characteristics of citizens being served; the external environment - for example, geography, climate, culture; the activities of other related agencies, both within and outside the public services; the quality of resources being used, including the capital stock; different accounting treatments; data errors; random (or idiosyncratic) fluctuation; different organizational priorities; differences in efficiency. 
With regard to the methodology used, the analysis reveals that around $45 \%$ of studies use the VRS (variable return to scale) input oriented method of DEA and the same percent use both VRS and CRS input oriented method. On the other hand, a quarter of the studies use SFA along with DEA, typically to regress factors of the efficiency scores in an attempt to determine influences on efficiency. COLS, translog analyses and Cobb-Douglas techniques are used in $25 \%$ of studies, also stochastic regression analysis and other parametric frontier techniques are used in $12 \%$ of the studies. Most of the results of the analysis highlight many divergent results between DEA and SFA methods and a small number of them reveal similar results of these techniques (Figure 9). A summary of studies comparing DEA and SFA and other parametric techniques is provided in the Appendix.

Figure 9. Shows here

\subsection{Overview of the efficiency measurements: DEA vs. SFA}

Table 1 in the Appendix presents the 21 articles reviewed. Considering that parametric methods, such as stochastic frontier analysis (SFA), use multivariate statistical methods to explore variations in output or costs between organizations and that non-parametric method, such as data envelopment analysis (DEA), attempt to measure efficiency by estimating the optimal level of output conditional upon the amount and mix of inputs, the articles were examined on the methodology used. The simulation studies reviewed include all possible pair-wise comparisons across the two relevant dimensions: non-parametric vs. parametric frontier estimation. In summary, the analysis reveals that both DEA and SFA have the potential to deliver biased estimates of inefficiency due to specification errors of one sort or another.

Characteristically, Giuffrida and Gravelle [15] use SFA, corrected ordinary least squares (COLS), and canonical regression as well as DEA on 90 UK FHSAs. COLS scores range from 0.868 to 0.915 , stochastic frontier scores range from 0.872 to 0.982 and canonical scores range from 0.80 to 0.81 , while DEA scores range from 0.904 to 0.994 . They conclude that the data nature and their availability influence the measurement of the efficiency and thus it is necessary to model the behavior which generates the data by choosing the appropriate mathematical form.

Similarly, Jacobs [16] uses SFA, OLS and DEA on a sample of up to 232 UK NHS hospital trusts. The OLS mean ranges from 0.541 to 0.611 , the SFA mean from 0.645 to 0.936 , and the DEA mean from 0.831 to 0.876 . The author concludes that the differences across methods may be due to noise and data deficiencies. Chirikos and Sear [17] using a sample of hospitals found SFA results ranging from 0.75 to 0.85 , concluding that DEA and SFA yield convergent results overall, but divergent 
results for individual hospitals, suggesting that hospital policymakers should carefully introduce these methods into the policy process. Rosenman [18] also concluded the same common views of what Chirikos [17] confirmed.

Bryce et.al [19] applied three models: DEA, a time varying SPF (stochastic production frontiers) and an enhanced FER (fixed-effects regression) in a sample of 585 HMOs, concluding on similar trends for the HMO industry as a whole and highlighting that these techniques are limited for either benchmarking or setting rates because the firms identified as efficient may be a consequence of model selection rather than actual performance. Likewise, Mortimer $[7,20]$ emphasized the need for real-world comparisons to determine the relative precision and policy value of DEA and SFA. Results of his analysis showed scores ranging from 0.83 to 0.86 in DEA and 0.81 to 0.86 in SFA models, suggesting that these two techniques employ quite distinct methodologies for frontier estimation and efficiency measurement, each with associated strengths and weaknesses, such that a trade off exists in selecting the correct approach.

The last 5 years Desai [21], Smith [22], Assaf [23], Liu [24] share the same prospect that neither DEA nor SFA can be regarded as clearly dominant, and that other mixed extended methods like quantile regression, or COLS can be used and likely can yield more reliable estimates, representing useful alternatives approaches in efficiency studies. Likewise, more recent studies, Kontodimopoulos [25], Martin [26], Veen [27], Nedela [28], suggest that SFA and DEA approaches along with other techniques are viable alternatives for analyzing the impact of environmental variables and dynamic effects on hospital cost efficiency, generating similar but more consistent results in empirical application to the efficiency analysis of healthcare units. Moreover, the majority of the researchers agree on the need of being aware of using both DEA and SFA methods, by checking the robustness of the impact of environmental variables on estimated efficiency.

\section{Discussion and Conclusions}

This review concentrates on selected efficiency studies using frontier efficiency measurement techniques. The aim was to locate results of combinations of frontier techniques (DEA, SFA) to evaluate healthcare efficiency. Thus the review was limited to search articles applying combined DEA and SFA with the aim of defining any differences on healthcare efficiency estimation and the reasons that are behind this. Therefore, this paper reviews empirical results drawn from published simulation studies.

The study of the 21 articles and the results, suggests that definitions of efficiency differ greatly depending on perspective, i.e., in the way that efficiency is defined as a relationship between what it costs and what service or outcome is received, rather than as a trait inherent in the provider. It is very important to address that the study seeks to focus on either the issue of extending the methods or introducing the results into the decision making process. Since the last review in this area [13,29], the number of studies which seek to measure health service efficiency and productivity has more than doubled. The inability to measure the real output of the healthcare industry, changes in health status and the low quality of available data still leads to problems. Thus, research in this area should still be reviewed cautiously and the results of studies interpreted carefully. The techniques are still criticized, but are continuingly being refined. However, estimated results may still be sensitive to changes in the basic assumptions or specifications of the models used and the 
characteristics of the environment in which the units operate. Consequently, the results may be valid only for the units under investigation, and not necessarily be generalized.

Overall, DEA and SFA were found to yield divergent efficiency estimates due to many factors such as statistical noise and inputs and outputs definition, as well as data availability. Nevertheless, different modelling approaches have advantages and disadvantages and the choice of the most appropriate estimation method should depend on the type of organizations under investigation, the perspective taken and the quality of the available data $[13,29]$. The issue of testing whether an environmental variable has a significant influence on the production process and any resulting efficiency estimates is recently overviewed. Jacobs [16], Smith and Street [30] note that the literature provides several different recommendations on how to handle such variables. From our review it is indicated that researchers, besides the combination of models to measure efficiency, introduce environmental variables in the analysis, aiming at better understanding the relationship of these factors to efficiency and thus at better decision making.

Given the limitations of frontier techniques at present it may be that they are best employed in tandem, when possible, and if different methods suggest similar directions for results then the validity of such findings is enhanced. Since the healthcare industry is one area where efficiency measurement may have a direct policy impact, a cautious approach is necessary. As well as refining methods, the means of making efficiency results useful in a practical setting needs careful attention. Although steps are being taken in this direction there is still some way to go. The use of models with restrictions placed upon the weight given to variables, in order to reflect underlying production models or policy values, is also an interesting area requiring further research to justify the use of such restrictions. There is still room for the use of more advanced methods bin efficiency measurement in the health and healthcare sector. The quality of data available for use may also be a problem to be addressed. Notwithstanding the caveats mentioned earlier regarding making comparisons across studies, and that perhaps work needs to be undertaken to think of ways of making efficiency studies comparable, these findings may have important policy implications for the organizational structure of healthcare delivery. To sum up, careful attention should be paid to the purpose of the analysis and to how results are to be used. In particular, if they are to be used to influence economic behavior - for example in the form of setting targets, or identifying candidates for inspection - then the potential costs of making incorrect inferences should be recognized.

\section{Acknowledgements}

The current paper constitutes part of the thesis titled "Evaluating Efficiency of Health Care Units with Stochastic Frontier Analysis". This research has been co-financed by the European Union (European Social Fund - ESF) and Greek national funds through the Operational Program "Education and Lifelong Learning" of the National Strategic Reference Framework (NSRF) - Research Funding Program: "Heracleitus II. Investing in knowledge society through the European Social Fund". Moreover, I would like to thank Dr Maria Katharaki, Visiting Lecturer at University of Athens for her useful scientific help and comments about the progress of this work. 


\section{References}

[1] IMF [International Monetary Fund]. Macro-Fiscal Implications of Healthcare Reform in Advanced and Emerging Economies, 2010.

[2] Oliveira M, Maisonneuve C, Bjornerud S. Projecting OECD health and long term care expenditures: What are the main drivers? OECD Working Paper No. 477, Paris.

[3] Cassel CK, Brennan TE.Managing medical resources: return to the commons? Journal of the American Medical Association 2007; 297(22):2518-21.

[4] Milstein A, Lee TH. Comparing physicians on efficiency. The new England Journal of Medicine 2007; 357(26):2649-52.

[5] The Leapfrog Group and Bridges to Excellence. Measuring Provider Efficiency Version 1.0. January 5, 2009. Available at http://www.google.com/url? sa=t\&source=web\&ct=res\&cd=4\&url=http\%3A\%2F\%2Fbridgestoexcellence.org\% 2Documents\%2FMeasuring Provider Efficiency Version1 12-3120041.pdf\&ei=BGdi SduROZWksAOjONyODQ\&usg=AFQjCNHFoeZecG $\quad 99$ bd3fmSFa EkBDAK K N A \&sig2=UoU5OCgfaXePw2Yp8h5xeA

[6] O'Kane M, Corrigan J, Foote SM, Tunis SR, Isham GJ, Nichols LM, Fisher ES, Ebeler JC, Block JA, Bradley BE, Cassel CK, Ness DL, Tooker J.Crossroads in Quality. Health Affairs 2008; 27(3):749-58.

[7] Mortimer D. A Systematic Review of Direct DEA vs SFA/DFA Comparisons. Centre for Health and Evaluation, Australia, 2002, Working Paper 136.

[8] Gong B, Sickles R. Finite sample evidence on the performance of stochastic frontiers and data envelopment analysis using panel data. Journal of Econometrics 1992; 51:259-284.

[9] Simar L, Wilson P.W. Estimation and inference in two-stage, semi-parametric models of production processes. Journal of Econometrics 2007;136:31-64.

[10] Newhouse J. Frontier estimation: how useful a tool for health economics? Journal of Health Economics 1994; 13: 317-322.

[11] Coelli T, Rao D, O'Donnell C, Battese G. An introduction to efficiency and productivity analysis (2nd ed.). New York 2005, Springer.

[12] Worthington A. Frontier Efficiency Measurement in Healthcare: A Review of Empirical Techniques and Selected Applications. Medical Care Research and Review 2004; 61(2):1-36.

[13] Hollingsworth B. Efficiency and productivity change in the English National Health service: Can data envelopment analysis provide a robust and useful measure? Journal of Health Services Research and Policy 2003;8.

[14] Hussey P, Vries H, Romley J, Wang M, Chen S, Shekelle P, McGlynn E. A Systematic Review of Health Care Efficiency Measures. Health Services Research 2009; 44(3): 784-805.

[15] Giuffrida A, Gravelle H.Measuring performance in primary care: Econometric analysis and DEA. Applied Economics 2001; 33 (2): 163-175. 
[16] Jacobs R. Alternative methods to examine hospital efficiency: Data Envelopment Analysis and Stochastic Frontier Analysis. Health Care Management Science 2001; 4:103-115.

[17] Chirikos T.N, Sear A.M. Measuring hospital efficiency: a comparison of two approaches. Health Services Research 2000; 34 (6):1389-1408.

[18] Rosenman R, Li T. Cost Inefficiency in Washington Hospitals: A Stochastic Frontier Approach Using Panel Data. Health Care Management Science $2001 ; 4: 73-81$

[19] Bryce C, Engberg J, Wholey D. Comparing the Agreement among Alternative Models in Evaluating Hmo Efficiency. Health Service Journal 2000;,35(2): 509528.

[20] Mortimer D. Methods for the Measurement of Hospital Efficiency: A Comparison of Frontier Estimation Techniques in a Sample of Victorian Public Hospitals. Department of Economics, Monash University: Unpublished Master of Economics (Honours) thesis, 2001.

[21] Desaia A, Ratick S, Schinnar A. Data envelopment analysis with stochastic variations in data. Socio-Economic Planning Sciences 2005;39:147-164

[22] Smith P, Street A. Measuring the efficiency of public services: the limits of analysis. Journal of the Royal Statistical Society: Series A (Statistics in Society) 2005;168: (2):401-417

[23] Assaf A, Matawie K. Cost efficiency modelling in healthcare food service operations. International Journal of Hospitality Management 2008; 27,(4):604613

[24] Lee R, Bott M, Gajewski B, Taunton R. Modelling Efficiency at the Process Level: An Examination of the Care Planning Process in Nursing Homes. Health Services Research 2009;44,(1):15-32

[25] Kontodimopoulos N, Papathanasiou N, Flokou A, Tountas Y, Niakas D. The Impact of Non-Discretionary Factors on DEA and SFA Technical Efficiency Differences. Journal of Medical Systems 2010;35,(5):981-989

[26] Martin S, Smith P. A comparison of English primary care trusts. Preliminary statistical analysis. The Health Foundation Inspiring Improvement 2010.

[27] Veen S. Comparative Efficiency Analysis from the Perspective of the Dutch Health Care Insurer. Determining the Usefulness of Efficiency Measures for Contracting Primary Care Organizations. Health Economics at the Erasmus University Rotterdam (Master Thesis), 2012

[28] Nedelea C, Fannin J. Efficiency Analysis of Rural Hospitals: Parametric and Semi-parametric Approaches. No 119725, Annual Meeting, Birmingham, Alabama from Southern Agricultural Economics Association 2012

[29] Hollingsworth $B$. The measurement of efficiency and productivity of health care delivery. Health Economics 2008;17 (10):1107-1128

[30] Jacobs R, Smith Pc, Street A. Measuring Efficiency in Health Care: Analytic Techniques and Health Policy, Cambridge, Cambridge University Press 2006. 
[31] Alshare K, Whiteside M.M. Stability analysis for DEA models: an empirical example. Academy of Information and Management Sciences Journal 2005;8 (2): 1-16.

[32] Ballestero E, Segura J. Objective measurement of efficiency: applying single price model to rank hospital activities. Computers and Operations Research 2004;31: 515-532.

[33] Banker R.D, Charnes A, Cooper W.W. Some Models for Estimating Technical and Scale Inefficiencies in Data Envelopment Analysis. Management Science 1984;30: 1078-1092.

[34] Charnes A, Cooper WW, Rhodes E. Measuring the efficiency of decisionmaking units. European Journal of Operational Research 1978; 2: 429-44.

[35] Charnes A, Cooper W.W. Preface to topics in Data Envelopment Analysis. Annals of Operations Research 1985; 2: 59-94.

[36] Coelli T, Prasada Rao, D. S, Battese G.E. An introduction to efficiency and productivity analysis (Boston, Kluwer Academic). Communication from the Commission to the Council and to the European Parliament. (COM/2006/0481 final) Efficiency and equity in European education and training systems \{SEC(2006) 1096\}.

[37] Cooper W, Seiford M, Zhu J. Handbook on Data Envelopment Analysis. Boston ( Kluwer Academic Publisher) 2004.

[38] Ferrier D, Valdmanis V. Rural hospital performance and its correlates. Journal of Productivity Analysis 1996; 7: 63-80.

[39] Fried O, Lovell A, Schmidt S, Yaisawarng S. Accounting for environmental effects and statistical noise in data envelopment analysis. Journal of Productivity Analysis 2002; 17(1-2): 157-174.

[40] Giokas D. The use of goal programming, regression analysis and data envelopment analysis for estimating efficient marginal costs of hospital services. Journal of Multi-Criteria Decision Analysis 2003;11 (4-5): 261 - 268.

[41] Katharaki M. Approaching the management of hospital units with an operation research technique. The Case of thirty two Greek Obstetric and Gynaecology Public Units. Health Policy 2008; 85 (1): 19-31.

[42] Katharaki M. A Data Envelopment Analysis model for measuring the efficiency impact of telemedicine on Greek obstetric and gynaecology services: Effects on individual hospital unit management. The Journal on Information Technology in Healthcare 2006; 4 (6): 373-383.

[43] Linna M. Measuring hospital cost efficiency with panel data models. Health Economics 1998; 7: 415-427.

[44] McKay L, Deily E, Dorner H. Ownership and changes in hospital inefficiency, 1986-1991. Inquiry 2003;39: 388-399.

[45] McNamara E. Welfare effects of rural hospital closures: a nested logit analysis of the demand for rural hospital services. American Journal of Agricultural Economics 1999; 81 (3): 686-91.

[46] Milstein A, Lee TH. Comparing physicians on efficiency. The new England Journal of Medicine 2007;357(26):2649-52. 
[47] Mutter L, Rosko D, Greene H, Wilson W. Translating frontiers into practice: taking the next steps toward improving hospital efficiency. Medical Care Research and Review 2011;68(1): 35-195.

[48] Nayar P, Ozcan A. Data envelopment analysis comparison of hospital efficiency and quality. J Med Syst 2008;32 (3):193-199.

[49] OECD. The Evaluation of Scientific Research: Selected Experiences (Paris: OECD 1997).

[50] OECD Education at a Glance. OECD Indicators 2005 (OECD Publishing).

[51] Ondrich J, Ruggiero J .Efficiency measurement in the stochastic frontier model. European Journal of Operational Research 2001;129:434-442

[52] Ozgen $\mathrm{H}$, Ozcan A. Longitudinal analysis of efficiency in multiple output dialysis markets. Health Care Management Science 2004; 7:253-261.

[53] Rosko D. Cost efficiency of U.S. hospitals: a stochastic frontier approach. Health Economics 2001; 10: 539-551.

[54] Rosko D, Mutter L. Inefficiency differences between Critical Access Hospitals and prospectively paid rural hospitals. Journal of Health Politics, Policy and Law 2010; 35(1): 95-126.

[55] Rosko D, Mutter L. What have we learned from the application of stochastic frontier analysis to U.S. hospitals? Medical Care Research and Review 2011; 68(1): 75S-100S.

[56] Rosko D, Proenca J. Impact of network and system use on hospital Xefficiency. Health Care Management Review 2005; 30: 69-79.

[57] Schmidt P, Sickles C. Production frontiers and panel data. Journal of Business and Economic Studies 1984; 2: 299-326.

[58] Sexton T, Leiken M, Sleeper S. The impact of prospective reimbursement on nursing home efficiency. Medical Care 1989; 27: 154-163.

[59] Thanassoulis E. Introduction to the theory and application of data envelopment analysis. A foundation text with integrated software (USA: Kluwer Academic Publishers 2001).

[60] Worthington A. An Empirical survey of frontier efficiency measurement techniques in education. Education Economics 2001; 9 (3): 245-268. 
Appendix

Table 1. Shows here 